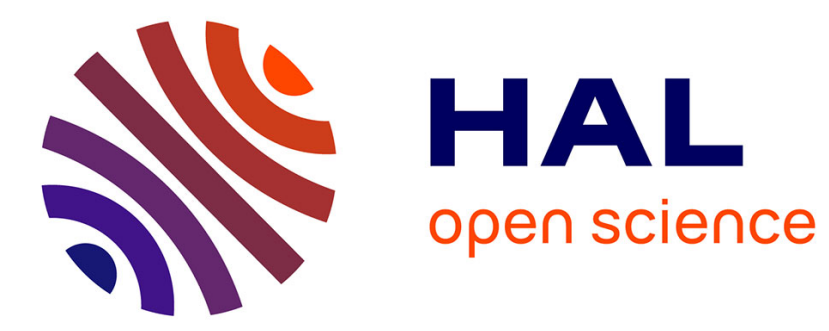

\title{
Moving from Digital Ecosystem to System of Information Systems
}

\author{
Majd Saleh, Marie-Helene Abel
}

\section{To cite this version:}

Majd Saleh, Marie-Helene Abel. Moving from Digital Ecosystem to System of Information Systems. IEEE 20th International Conference on Computer Supported Cooperative Work in Design (CSCWD), , May 2016, Nanchang, China. pp.91-96, 10.1109/CSCWD.2016.7565969 . hal-01396304

\section{HAL Id: hal-01396304 https://hal.science/hal-01396304}

Submitted on 14 Nov 2016

HAL is a multi-disciplinary open access archive for the deposit and dissemination of scientific research documents, whether they are published or not. The documents may come from teaching and research institutions in France or abroad, or from public or private research centers.
L'archive ouverte pluridisciplinaire HAL, est destinée au dépôt et à la diffusion de documents scientifiques de niveau recherche, publiés ou non, émanant des établissements d'enseignement et de recherche français ou étrangers, des laboratoires publics ou privés. 


\title{
Moving from Digital Ecosystem to System of Information Systems
}

\author{
Majd Saleh, Marie-Hélène Abel \\ Sorbonne Universités, Université de \\ Technologie de Compiègne, CNRS, \\ UMR 7253 Heudiasyc \\ Compiègne, France \\ Email: \{majd.saleh,marie-helene.abel\}@utc.fr
}

\begin{abstract}
Digital Ecosystem (DE) is a concept emerged from the natural existence of business ecosystem, which in turn is taken from the concept of biological ecological systems. On the other hand, System of Information Systems (SoIS) are special type of System of Systems (SoS) that deals with several Information Systems producing overwhelming amount of information. In this paper we aim to define the concepts of DE, SoS, and SoIS. Then, provide the guidance for moving from Digital Ecosystem to System of Information Systems. Thus, we aim to draw the link between the model of DE presented in literature and the concept of SoS and SoIS. Then, we propose an architectural model of System of Information Systems (SoIS) and investigate the knowledge base role in such complex system operating in the environment of a Digital Ecosystem. These complex Digital Ecosystems pose a significant technical improvement in terms of information interoperability that overcomes conceptual and technical barriers. In this paper we are moving from Digital Ecosystem to System of Information Systems by defining the similarities between the two concepts, then proposing an architectural model of SoIS in correspondence to DE model.
\end{abstract}

\section{INTRODUCTION}

Information Systems have begun to turn simplicity to complexity, and to turn hard-to-use to easy-to-use. However, effectiveness and efficiency on information systems is not satisfying [1]. This universal nature of existing Information Systems has generated a strong interest in using an existing set of systems as the basis for a System of Systems [2], in a way these systems work and communicate with each other in order to aggregate services and produce new ones emerged from existing systems. Therefore, the competitive advantage is no longer achieved only through efficiency, quality, and quick responsiveness. While each of these factors is important, the ability to employ available solution to the favor of solving emergent problems is of great importance. This can be achieved by aggregating services of existing Information Systems and providing centralized access to these service. In turn, this makes innovation, flexibility, coordination, integration, and speed the new success factors of todays work environment. Those factors can be reached through the concept of System of Information Systems (SoIS).

On the other hand, a Digital Ecosystem is a distributed, adaptive, open socio-technical system with properties of selforganization, scalability and sustainability inspired from natural ecosystems. Digital Ecosystem models are informed by knowledge of natural ecosystems, especially for aspects related to competition and collaboration among diverse entities [3]. As a research area, such as DE and SoIS, matures and starts to receive attention, it becomes important to harness its benefits to solve occurring problems in todays technical world. The purpose of this paper is to investigate the similarity between DE and SoIS in order to propose an architecture of a System of Information Systems comprised of independent Information Systems working in the environment of a Digital Ecosystem.

This paper is organized as follows: in section 2 we will define the concept of Digital Ecosystem and highlight our findings in this field from a previous study. Then, the notion of System of Systems is defined and a discussion of the similarities between System of Systems and Digital Ecosystem is presented to draw the links between these two concepts. After that, in section 4, we define the concept of SoIS and present an architectural model of it. Then we present an example following our architectural model of SoIS. Later, we discuss our findings in section 5. Finally, we conclude with section 6 .

\section{Digital ECOSYSTEM}

In this section we go through the definition of Digital Ecosystem and its origin. Then, we summarize our findings from a previous systematic mapping of studies in the field of Digital Ecosystem. The systematic mapping can provide indicators on the trends in the field of Digital Ecosystem, and how studies view Complex Digital Ecosystems such as System of Systems (SoS).

\section{A. Definition}

Digital Ecosystem is defined by Digital Ecosystem and Business Intelligence Institute (DEBII) from the perspective of specialization, which sees it as "an open, loosely coupled, domain clustered, demand-driven, self-organizing, and agent based environment which each species is proactive and responsive for its own benefit and profit" [4].

The concept of digital ecosystem itself was adopted from the concept of business ecosystem. [5] defines business ecosystem and the idea behind the adoption of the term "ecosystem" as follows: "The term [ecosystem] circumscribes the microeconomics of intense coevolution coalescing around innovative ideas. Business ecosystems span a variety of industries. The companies within them co-evolve capabilities around innovation and work cooperatively and competitively to support new products, satisfy customers needs and incorporate the next 
round of innovation." For [6] a business ecosystem is a loose network of suppliers, distributors, outsourcing firms, market of related products or services, technology providers, and a host of other organization. All of them affect and are affected by the creation and delivery of companys own offerings. According to [6] also, these entities share the fate of the network as a whole, regardless of the members apparent strength.

\section{B. Summary of the systematic mapping of studies in the field of Digital Ecosystem}

In a previous study [7] we reviewed research materials in the field of Digital Ecosystem obtained from different databases. The results of the selection process of the research materials are presented as follows: In the first step, five databases were searched individually (ACM, Elsevier, IEEE Xplore, Science Direct, and Springer) using the following keywords (Digital Ecosystems, System of Systems, Business Ecosystem, Collaboration Network, and Social Media). A total of 26,763 studies were found. In the second step, 186 of the 26,763 studies were selected based on a search on the title and abstract. The first step set the search domain to the area of interest, and the second step filtered the studies based on the title and abstract. If we were to perform the search on each title and abstract directly, the results would be different and some of the results might not be targeting our area of interest. A search for title and abstract in a database that holds more than million studies is not as specific as a search of a few thousands studies. In the third step, eleven duplicate studies were excluded resulting in 175 studies. In the fourth step, the detailed inclusion and exclusion criteria, can be found in [7], were applied and resulted in 31 studies.

The 31 studies selected for systematic mapping were classified based on the six different topics (i.e., Business Ecosystem, Collaboration Systems, Digital Business Ecosystem, Digital Ecosystem, Semantic Web, Social Ecosystem, Social Media Network, and System of Systems), and mapped with respect research goals. A map was developed in the form of bubble charts. The mapping is shown in [7] with detailed discussion. It maps the number of studies addressing each topic with respect to the goal of the research conducted in these studies.

It is notable from the bubble chart the lack of studies concerning topic of System of Systems, and how it corresponds to the notion of Digital Ecosystem. In the following section, we define what is System of Systems, and how it can be considered as a complex Digital Ecosystem.

\section{SySTEM OF SySTEMS}

\section{A. Definition}

The notion of System of Systems (SoS) can be viewed as an evolution of the standard notion of systems. The act of aggregating systems leads to the creation of new forms of systems. This may reveal additional features that add complexity to the description of these systems and may be referred to as System of Systems.

As many definitions of a SoS exist, we can summarize the definitions mentioned in [8] [9] [10] [11]. In the light of the definitions mentioned in the literature, it is possible to describe the notion of System of Systems (SoS) as the following;
Systems of systems are large-scale integrated systems which are heterogeneous and independently operable on their own, but are networked together for a common goal. The goal, as mentioned before, may be cost, performance, robustness, etc. In other terms, a System of Systems is a super system comprised of other elements which themselves are independent complex operational systems and interact among themselves to achieve a common goal. Each element of a SoS achieves wellsubstantiated goals even if they are detached from the rest of the SoS.

\section{B. System of Systems as Digital Ecosystem}

In order to understand how System of Systems are related to Digital Ecosystems, we first need to know how Digital Ecosystems are modeled. [4] aims to solve the conceptual ambiguity and help researchers better understand what DE is by employing ontology to represent the conceptual model of DE. This study views DE in terms of concepts and its species alongside with the parts that composes them. The relation between those species as composition and generalization links are also represented. In addition, this study shows how all the species composing the DE work altogether in a digital environment, and take care of this environment as it is a mutual interest for them.

Based on this model we can view the System of Systems as a Digital Ecosystem, where different systems of the SoS can be considered as species in the DE. Also base on the model of $\mathrm{DE}$, one of the systems composing SoS can play the role of the leader system as we will see in the model presented in (Fig. 1) and then the example provided in the following section about MEMORAe SoIS. Furthermore, the resemblance between SoS and $\mathrm{DE}$ can be found in the description of DE by [12]. DE may be described as a network of niches, interconnected with or via the platform, suited to the co-evolution of stakeholders. Similarly, [11] defines SoS as a set of different elements connected or related so as to perform a unique function not performable by the elements alone. These elements (systems) are the network of niches in the DE as mentioned in the previous description.

The track of this study is focused on the System of Information Systems as a special type of SoS. Similar to SoS, SoIS can be considered as Digital Ecosystems where Information Systems takes the role of species (or network of niches) in the DE model. To put the idea of why we need SoIS in perspective, let us take the following scenario; Say a group of journalists are investigating a certain topic. First they might visit various Social Media Networks and search for this topic and the people involved in it. Next, they might need to unveil the ambiguity on certain aspects to better understand the topic, so one of them starts using Wikipedia [13] or DBpedia [14] to search for keywords related to this topic, while the others use the archive system from other databases to search for information. Later on, they might publish some of their findings on various Social Media Networks to see the public reaction to the topic under investigation. Of course, not to forget that they need to store and keep track of the information they collected from different Information Systems, the activities of each one of them to assign credit of information, and be able to access the information at all times to reform an encompassing idea about the topic at hand. To sum things up, we mentioned a scenario 


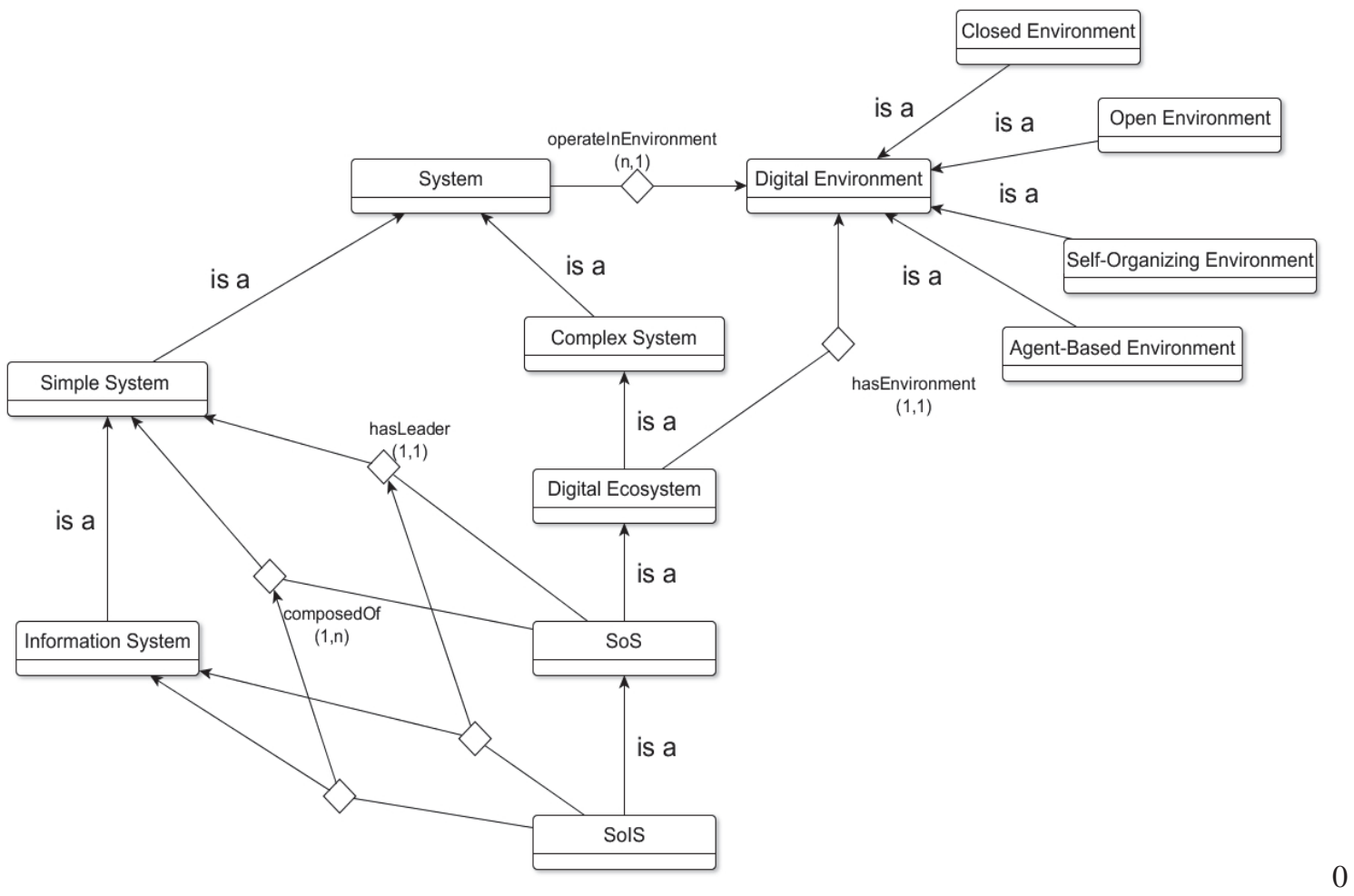

Fig. 1. Model to show the relation between SoIS and DE, and the components of SoIS.

where several users surfed several Information Systems and collected information from those systems separately. Each user also had to work with the information collected from separate systems collectively. The struggle is with accessing all these different Information Systems and keeping track of the information obtained and created while doing the work required. The problems we face here reside in the time required to traverse between different systems and keep track, organize, and archive all the information obtained from these systems. Hence, we move towards a solution in the shape of a Digital Ecosystem. The SoIS components are considered to be the species in this DE, and the network in which the SoIS operates is the environment of the DE.

The model presented in figure (Fig. 1) shows the relation between DE and SoS, and hence, SoIS. It also show how SoIS is composed of the leader system and other Information Systems. The relations in this model represent, alongside the name of the relation, the number of instances of each concept that can be associated with the relation like One-to-One, or Many-to-One.

\section{System OF INFORMATION Systems}

In this section, we will go through the definition of SoIS. Then, we will present an architectural model for SoIS. Finally we propose an intial designs for the SoIS that can serve as an example of the architecture.

\section{A. Definition}

The notion of System of Information Systems is defined by [15] as "networks of agents interacting in a specific technology area under a particular institutional infrastructure for the purpose of creating, diffusing, and utilizing technology focused on knowledge, information, and competence flow." [16] describe SoIS as "the specific clusters of the firms, technologies, and industries involved in the generation and diffusion of new technologies and in the knowledge flow that takes place among them."

Based on the definitions provided we can summaries the features of SoIS as follows:

- SoIS addresses the impact of the interrelationships between different IS.

- SoIS is concerned with the flow of information and knowledge among different information systems.

- SoIS is responsible for generating information from the emergent IS.

- Information interoperability is a key issue when designing a SoIS.

\section{B. Architecture}

The generic architecture of the SoIS is presented in this section. As seen in (Fig. 2), the SoIS will aggregate services from several Information Systems (System A, System B etc.). These systems are working separately. Each of which has its own services and databases. The services of these systems are denoted as solid rectangles inside the system (Service 1A, Service 1B etc.). Information can be represented in different ways within different systems, thus, the problem of accessing information from different systems. Therefore, there is a growing need for a solution to this interoperability issue. A solution 


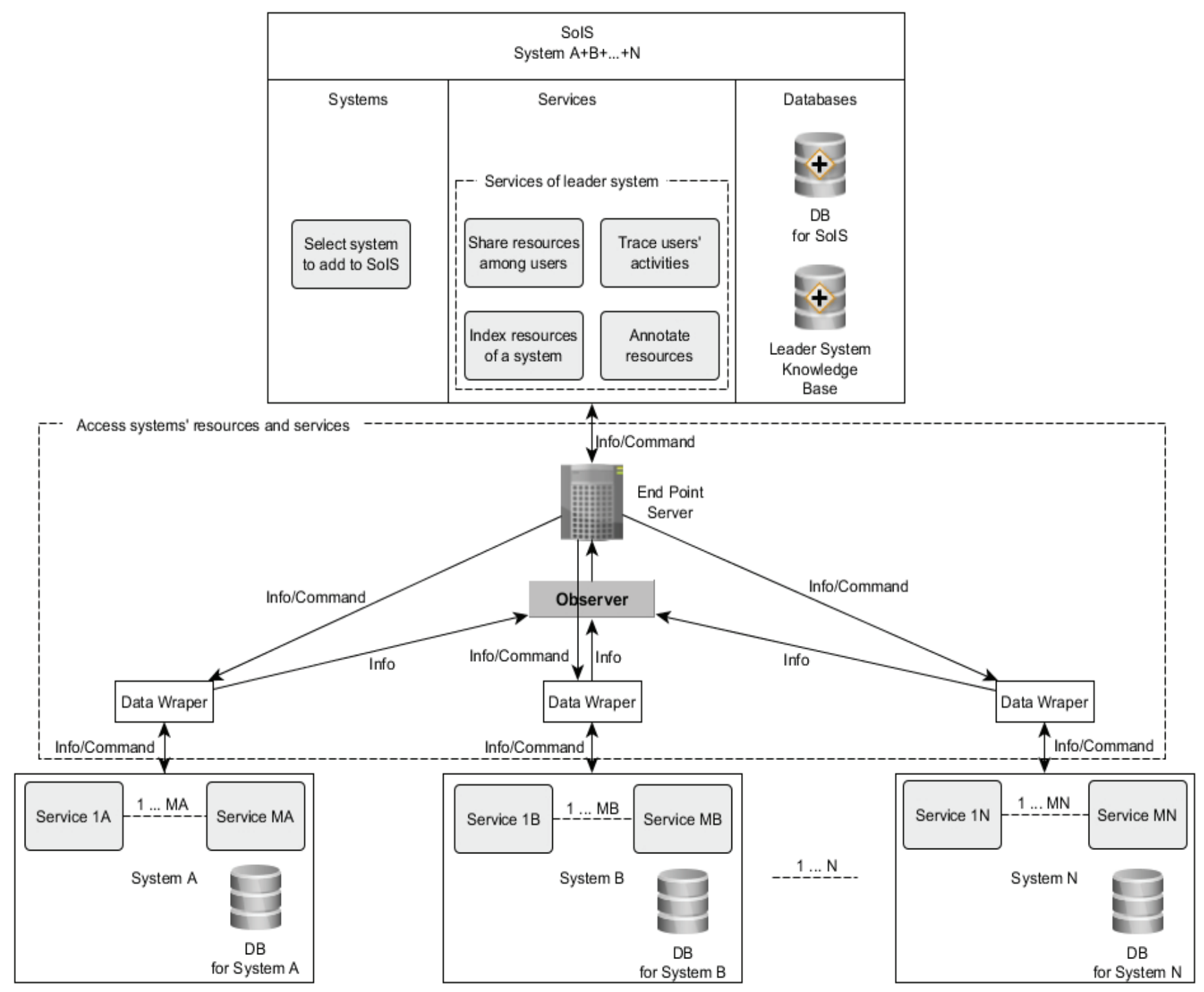

Fig. 2. Architectural model of the SoIS.

to the problem of assuring interoperability within the SoIS is to control the communication medium among the systems. Two methods, outlined by [17], include:

- Creating a software model of each system, where the software model collects data from the system and generates the outputs.

- Creating a common language to describe data, where each system can represent its data such that other systems may interpret.

Due to overhead restrictions on a common language, it is not often that standard language for data interpretation is used throughout the SoIS. Therefore, creating individual software models for the various systems is the most widely used approach to ensure interoperability within a SoIS. This can explain the need for data wrapper as a mediator software between the SoIS and its component systems. The SoIS is represented as a group of systems connectors, services and databases. The services residing in the SoIS can either be utilization of existed services from the Information Systems comprising the SoIS, or emerging services created from the aggregation of different Information Systems.

The ideas present in this architecture of SoIS can be summarized in the following list:

- The user is provided with access to several Information Systems.

- The user can choose which Information System(s) he/she would like to connect to.

- After connecting to various systems of choice, the user can access resources and services in their respective environment.

- It is then possible to work with resources produced by different Information Systems from within the SoIS.

- The resources produced by different Information Sys- 
tems are managed in the SoIS by the means of the services provided by the leader system.

- Using the services of the leader system as a way to manage knowledge with the SoIS will enable the user from indexing, sharing and tracing resources within the SoIS.

\section{MEMORAeSoIS}

In this section we propose a prototype developed based on the architectural model presented earlier. As mentioned in the architecture of SoIS, leader system plays a leading role in the orchestration of SoIS as a knowledge base serving all other Information Systems. We propose MEMORAe system to play the role of the leader system in the SoIS. So, what is MEMORAe?

As defined by [18], MEMORAe approach aims to manage heterogeneous information resources within organizations. The approach is comprised of a semantic model (called MEMORAe-core 2) and a web platform (called MEMORAe) which is based on the semantic model. The model and the platform make together a support to enhance the process of organizational learning.

The MEMORAe project uses the Semantic Web standards, therefore, the ontologies used in the system are written in OWL. Users registered in MEMORAe system can access one or more knowledge bases. When a base is chosen, a user can view a semantic map of concepts related to the selected base. Then, a user can create and share resources around the concepts of the map.

The user of MEMORAe web platform is able to create various resources within the platform. These resources include documents, notes, clusters of notes, weblinks, contacts, events, tours, and forums. The user is also able to index these resources using the semantic map visualized as concepts and instances in MEMORAe. Furthermore, MEMORAe gives users the ability to annotate these resources and share them within different sharing spaces, in addition to tracing users' activities within the platform. The limitation here is in the resources available inside MEMORAe. While the platform permits creating and managing various types of resources, all of them are native to the platform. Users might benefit more if the capabilities provided by MEMORAe approach (indexing, annotating, sharing, tracing the resources) is applicable to resources created by other Information Systems in the work scope of the user but outside the scope of MEMORAe. Such resources might be emails, wiki pages, social media entries, and others related to users' work. Hence, we propose to develop a prototype based on the architecture of SoIS that puts MEMORAe approach as a leader system among other Information Systems in this Digital Ecosystem. That means taking MEMORAe web platform and evolve it into a MEMORAe SoIS. This can be developed following the SoIS architecture presented earlier with MEMORAe as a leader system and other Information Systems as composite systems of this SoIS. By doing so, users are given a wider work scope with the following features:

- Access resources coming from different Information Systems in a centralized location.
- Create resources using a dedicated Information System. These resources can be accessed either from the MEMORAe SoIS or the dedicated system where they are created.

- Organize resources around a semantic map.

- Share resources within different sharing spaces.

- Annotate resources in order to highlight certain ideas related to the resources.

- $\quad$ Record the users activities inside MEMORAe SoIS.

\section{Discussion}

In this section we will elaborate on the role of MEMORAe System as a knowledge base in the orchestration of SoIS and a leader system in the Digital Ecosystem. Furthermore, we will highlight the importance of its features in providing the added value for the SoIS.

In the Example of SoIS presented earlier we can view MEMORAe system as a knowledge base that handles storing, organizing, and sharing of resources from different Information Systems. MEMORAe system aims, by its design, to facilitate knowledge sharing and capitalization within those different systems. All types of resources are indexed by a semantic map that represents the concepts of interest for the user [19]. furthermore, The semantic map defines a common reference shared between all users. The focus concept of the semantic map is a concept in which the user is interested in. The shared resources are indexed by the concepts of the semantic map. In this orchestration of SoIS, MEMORAe is introduced to new resources produced by Information Systems that reside outside its scope, hence the use of MEMORAe as it supports different types of social resources (wiki, forums, chat) and documentary resources (documents, images, web link). This constitutes an added value for the Information Systems comprising the SoIS, as data produced from thoes systems is capitalized as knowledge within MEMORAe.

User activity traces and recommendation system is another feature of MEMORAe that emphasize on the role of a knowledge base within a SoIS. MEMORAe systems owns a mechanism that models, records, and analyzes users traces. It allows evaluating competencies for recommending users with more expertise on certain subjects for either personal purpose or to an entire group of users [20]. Additionally to the previous point of MEMORAe capitalizing knowledge from different Information Systems, users' activities from those systems are also capitalized by using MEMORAe as a knowledge base in the SoIS.

The architectural model of SoIS is linked to Digital Ecosystem model presented earlier in this study. The leader system along with other Information systems can be considered as species in the Digital Ecosystem. Furthermore, the network where the endpoint server operates with the data wrappers responsible for data interoperability are considered to be the environment of the Digital Ecosystem.

\section{CONCLUSION}

Our goal was to investigate the similarity between System of Systems and Digital Ecosystems by defining and modeling 
the two concepts, and checking the resemblance between them. The reason we did that, is to help us take current Information Systems and move towards System of Information Systems (SoIS) to aggregate services and manage the process of exchanging information with simplicity and ease in a Digital Ecosystem environment. To achieve our goals, we undertake this research with a link to a previous systematic mapping of studies in the field of Digital Ecosystem. Based on the systematic mapping we presented a model of Digital Ecosystem and link this model to the concept of Systems of Systems. Then we moved to define a special type of SoS which is the System of Information Systems. The aim was focused towards investigating the knowledge base role in a System of Information Systems (SoIS), and providing an architectural model of SoIS to guide the migration for such complex Digital Ecosystem. Finally, we presented an example of a SoIS composed of various Information Systems with MEMORAe approach in its core as a system to manage the resources produced by different systems comprising the SoIS. We found potentials in deploying MEMORAe approach to manage the resources produced by different systems in the SoIS. It was also clear that combining resources from various Information Systems and manage them within a knowledge base will result in an added value to users not present when those systems were operating separately out side the envoronment of the Digital Ecosystem.

The next step is to expand our work and introduce new Information Systems to the SoIS based on the example presented in this study and users needs. It should also be tested in a real world experiment. The SoIS should keep simple interface, with all the services as far from the user as a single click, to keep the users experience useful and friendly.

\section{ACKNOWLEDGMENT}

This project is done under ECOPACK project and funded by ANR-ASTRID programme.

\section{REFERENCES}

[1] H. An, "Research on information systems problems based on an ecological angle," in Challenges in Environmental Science and Computer Engineering (CESCE), 2010 International Conference on, vol. 1. IEEE, 2010, pp. 281-284.

[2] J. J. Simpson and C. H. Dagli, "System of systems: Power and paradox," in System of Systems Engineering, 2008. SoSE'08. IEEE International Conference on. IEEE, 2008, pp. 1-5.

[3] G. Briscoe and P. De Wilde, "Digital ecosystems: evolving serviceorientated architectures," in Proceedings of the 1st international conference on Bio inspired models of network, information and computing systems. ACM, 2006, p. 17.

[4] H. Dong and F. K. Hussain, "Digital ecosystem ontology," in Emerging Technologies and Factory Automation, 2007. ETFA. IEEE Conference on. IEEE, 2007, pp. 814-817.

[5] J. F. Moore, The death of competition: leadership and strategy in the age of business ecosystems. HarperBusiness New York, 1996.

[6] M. Iansiti and R. Levien, "Strategy as ecology," Harvard business review, vol. 82, no. 3, pp. 68-81, 2004.

[7] M. Saleh, M.-H. Abel, and V. Misseri, "Investigating the similarity between collaboration systems and digital ecosystems," in Computer Supported Cooperative Work in Design (CSCWD), 2015 IEEE 19th International Conference on. IEEE, 2015, pp. 30-35.

[8] M. Jamshidi, System of systems engineering: innovations for the twentyfirst century. John Wiley \& Sons, 2011, vol. 58.
[9] P. G. Carlock and R. E. Fenton, "System of systems (sos) enterprise systems engineering for information-intensive organizations," Systems engineering, vol. 4, no. 4, pp. 242-261, 2001.

[10] W. H. Manthorpe, "The emerging joint system of systems: A systems engineering challenge and opportunity for apl," Johns Hopkins APL Technical Digest, vol. 17, no. 3, p. 305, 1996.

[11] E. Rechtin and M. W. Maier, The art of systems architecting. CRC Press, 2000.

[12] A. Gawer and M. A. Cusumano, Platform leadership: How Intel, Microsoft, and Cisco drive industry innovation. Harvard Business School Press Boston, 2002.

[13] Wikipedia, "Wikipedia, the free encyclopedia," 2015, [Online; accessed 20-May-2015]. [Online]. Available: urlhttp://en.wikipedia.org/

[14] DBpedia, "Dbpdia," 2015, [Online; accessed 20-May-2015]. [Online]. Available: urlhttp://wiki.dbpedia.org/

[15] B. Carlsson and R. Stankiewicz, "On the nature, function and composition of technological systems," Journal of evolutionary economics, vol. 1, no. 2, pp. 93-118, 1991.

[16] S. Breschi and F. Malerba, Sectoral innovation systems: technological regimes, Schumpeterian dynamics and spatial boundaries. Centro studi sui processi di internazionalizzazione, Università commerciale'Luigi Bocconi', 1996.

[17] R. M. Bowen and F. Sahin, "A net-centric xml based system of systems architecture for human tracking," in System of Systems Engineering (SoSE), 2010 5th International Conference on. IEEE, 2010, pp. 1-6.

[18] C. M. Ala Atrash, Marie-Hélène Abel, "Supporting organizational learning with collaborative annotation," in 6th international conference on knowledge management and information sharing (KMIS), 2014, pp. 237- 244.

[19] A. Atrash, M.-H. Abel, C. Moulin, N. Darène, F. Huet, and S. Bruaux, "Note-taking as a main feature in a social networking platform for small and medium sized enterprises," Computers in Human Behavior, 2015.

[20] N. Wang, M.-H. Abel, J.-P. Barthes, and E. Negre, "Mining user competency from semantic trace," in Computer Supported Cooperative Work in Design (CSCWD), 2015 IEEE 19th International Conference on. IEEE, 2015, pp. 48-53. 